\title{
The cholecystokinin-A receptor mediates inhibition of food intake yet is not essential for the maintenance of body weight
}

\author{
Alan S. Kopin, ${ }^{1}$ Wendy Foulds Mathes ${ }^{2}$ Edward W. McBride, ${ }^{1}$ Minh Nguyen, ${ }^{1}$ \\ Wisam Al-Haider, ${ }^{1}$ Frank Schmitz, ${ }^{1}$ Susan Bonner-Weir, ${ }^{3}$ Robin Kanarek, ${ }^{2}$ \\ and Martin Beinborn ${ }^{1}$ \\ ${ }^{1}$ Department of Medicine, GRASP Digestive Disease Center, Tupper Research Institute, New England Medical Center, \\ Tufts University School of Medicine, Boston, Massachusetts 02111, USA \\ ${ }^{2}$ Department of Psychology, Tufts University, Medford, Massachusetts 02155, USA \\ ${ }^{3}$ Joslin Diabetes Center, Harvard Medical School, Boston, Massachusetts 02215, USA
}

Address correspondence to: Alan S. Kopin, Tupper Research Institute, 750 Washington Street, Box 239, Boston, Massachusetts 02111, USA. Phone: (617) 636-7703; Fax: (617) 636-8692; E-mail: alan.kopin@es.nemc.org

Received for publication August 12, 1998, and accepted in revised form December 17, 1998.

\begin{abstract}
Food intake and body weight are determined by a complex interaction of regulatory pathways. To elucidate the contribution of the endogenous peptide cholecystokinin, mice lacking functional cholecystokinin-A receptors were generated by targeted gene disruption. To explore the role of the cholecystokinin-A receptor in mediating satiety, food intake of cholecystokinin-A receptor ${ }^{-/-}$mice was compared with the corresponding intakes of wild-type animals and mice lacking the other known cholecystokinin receptor subtype, cholecystokinin-B/gastrin. Intraperitoneal administration of cholecystokinin failed to decrease food intake in mice lacking cholecystokinin-A receptors. In contrast, cholecystokinin diminished food intake by up to $90 \%$ in wild-type and cholecystokinin-B/gastrin receptor ${ }^{-/}$mice. Together, these findings indicate that cholecystokinin-induced inhibition of food intake is mediated by the cholecystokinin-A receptor. To explore the long-term consequences of either cholecystokinin-A or cholecystokinin-B/gastrin receptor absence, body weight as a function of age was compared between freely fed wild-type and mutant animals. Both cholecystokinin-A and cholecystokinin-B/gastrin receptor-/- mice maintained normal body weight well into adult life. In addition, each of the two receptor ${ }^{-/-}$strains had normal pancreatic morphology and were normoglycemic. Our results suggest that although cholecystokinin plays a role in the short-term inhibition of food intake, this pathway is not essential for the longterm maintenance of body weight.
\end{abstract}

J. Clin. Invest. 103:383-391 (1999).

\section{Introduction}

Cholecystokinin (CCK) is a peptide hormone and neurotransmitter that was first postulated to regulate feeding behavior 25 years ago (1). It has since been shown in a variety of vertebrate species including goldfish, mice, rats, dogs, sheep, pigs, monkeys, and humans that administration of CCK reduces food intake (2-9). Subsequent analyses using subtype selective agonists have suggested that the decrease in food consumption resulting from exogenous cholecystokinin is mediated by the CCK-A receptor (CCK-AR) (7-9). These investigations also suggested that stimulation of the other known CCK receptor subtype, CCK-B/gastrin (CCK-BR), plays no significant role in the inhibition of food intake resulting from exogenously administered peptide.

The development of receptor subtype specific antagonists provided additional tools (10) to explore the role of both exogenous and endogenous cholecystokinin in feeding behavior. Multiple studies have demonstrated that the CCK-AR-specific antagonist L-364,718 blocks the inhibition of food consumption resulting from exogenously administered CCK-8 (7-9). Furthermore, experiments with L-364,718 provided support for the physio- logic significance of endogenous CCK-8 as a mediator of feeding behavior. Administration of L-364,718 alone to mice, rats, pigs, or monkeys increases food intake when assessed using a variety of feeding paradigms (7-9, 11-13). In addition, it was reported that L-364,718 enhanced hunger ratings in humans (14). Despite the large body of evidence in support of endogenous CCK as a physiologic mediator of food intake, there are a considerable number of studies that have failed to detect a significant increase in feeding after L-364,718 administration $(3,7,8,15,16)$. The physiologic variables and/or subtle differences in the feeding paradigms, which explain the discrepant findings with this CCK-AR-specific antagonist are currently unknown.

The understanding of endogenous cholecystokinin as a modulator of food intake was further complicated by results obtained with L-365,260, a CCK-BR selective antagonist. The potency of L-365,260 in stimulating food intake, as well as in delaying the postprandial satiety sequence in partially satiated rats was reported to exceed that of L-364,718 (17). Although controversial (8, 18-21), this finding led to the hypothesis that under certain conditions, the CCK-BR also plays a role in modulating food 
intake. As a possible mechanism, it was postulated that during stress or anxiety, CCK-BR-mediated pathways are activated and in turn may influence eating behavior $(7,9)$.

Pharmacologic manipulations, despite their inherent limitations, have provided much insight into how exogenous and/or endogenous CCK affects short-term food intake. However, it has been difficult using a parallel approach to clarify the physiologic relevance of endogenous CCK in the long-term control of body weight. As an alternative strategy, targeted gene disruption has emerged as a powerful tool for assessing the role of a particular gene product in the maintenance of body weight and/or the development of obesity. Inactivation of either the $\beta 3$-adrenergic receptor (22), the serotonin $2 \mathrm{C}(23)$, the gastrin-releasing peptide 3 (24), or the melanocortin4 (25) receptor led to mice with an increase in body weight relative to normal controls, thereby suggesting an essential role of each of these pathways.

It is of note that the results obtained with targeted gene disruption have not always been those predicted on the basis of pharmacologic manipulations. Although central administration of glucagon-like peptide 1 decreases food intake (26), deletion of the corresponding receptor does not lead to obesity in mice (27). Along the same theme, targeted gene disruption of neuropeptide $Y$, although well established as a central stimulator of food intake, did not result in mice with altered body weight (28). Pharmacologic investigations and gene targeting may therefore be viewed as complementary approaches in establishing the physiologic significance of a given pathway.

Interest in the potential role of the CCK-AR in regulating satiety and body weight was enhanced by a recent report that an inbred line of rats, Otsuka Long-Evans Tokushima Fatty (OLETF), lack CCK-A receptors because of a spontaneous deletion of the corresponding gene $(29,30)$. With aging, these animals develop obesity, diabetes, and pancreatic islet fibrosis with a strong male predominance of the phenotype. It is, however, not clear whether these abnormalities result from absence of the CCK-AR, because OLETF rats appear to carry additional genetic defects $(31,32)$.

One advantage of targeted gene disruption is that resulting abnormalities can be attributed to the absence of a specific gene product. We have, therefore, generated mice lacking CCK-A receptors which, together with previously reported CCK-BR knockout mice $(33,34)$, provide novel in vivo models to assess the mechanisms underlying the CCK-mediated regulation of food intake. In this study, we examine the relative contributions of the CCK$\mathrm{A}$ and the CCK-B receptors in modulating feeding behavior, as well as assess the role of endogenous CCK in determining body weight over a prolonged period of time.

\section{Methods}

Animals. 129/SvEv mice were obtained from The Jackson Laboratory (Bar Harbor, Maine, USA) and housed in microisolator cages. Approval for animal experimentation was obtained from the Tufts-New England Medical Center Animal Research Committee. Mice were housed at a maximum of eight animals per cage in a temperature and humidity controlled room $\left(21^{\circ} \mathrm{C}\right.$, $30 \%$ ) with a 12 -h light/12-h dark cycle (lights on at 0800 ). For restricted feeding and conditioned taste aversion studies, mice were individually housed in standard plastic cages.
Peptides. Sulfated cholecystokinin octapeptide, CCK-8 (Peninsula Laboratories Inc., San Carlos, California, USA), was diluted in Dulbecco's PBS (GIBCO BRL, Rockville, Maryland, USA) before intraperitoneal injection. CCK-8 was administered in a volume of $10 \mathrm{ml} / \mathrm{kg}$ body weight; dosing of peptide was adjusted by altering the concentration. Iodinated CCK-8 (specific activity 2,200 Ci/mmol) was obtained from NEN Life Science Products (Boston, Massachusetts, USA).

Construction of the CCK-AR targeting vector. The human CCK-AR cDNA was obtained by PCR based on a published sequence (35, 36). The human cDNA was used as a hybridization probe to screen a mouse PCC4 genomic library (Stratagene, La Jolla, California, USA). Two overlapping genomic phage clones were isolated. Restriction enzyme analysis and limited DNA sequencing were used to determine that the genomic fragment included exons $2-5$ of the CCK-AR gene $(37,38)$.

In the targeting construct (Fig. 1), exon 3 sequence encoding a portion of the third transmembrane domain and the second intracellular loop including the "ERY" motif (39) was replaced with the neomycin resistance gene under the control of the thymidine kinase promoter (pMC1Neo; Stratagene) (Fig. 1). A CCK-A receptor without these domains will bind neither ligand nor signal (Kopin, A., and Beinborn, M., unpublished data). The insertion of the neomycin resistance gene within the proteincoding region of the receptor further ensured that the targeted CCK-A receptor gene would not produce a functional protein.

Gene targeting in embryonic stem cell. The CCK-AR gene targeting vector was linearized with Xma I and electroporated into R1 embryonic stem cells (ES; gift of A. Nagy, Mount Sinai Hospital, Toronto, Ontario, Canada) $(33,40)$. G418-resistant ES cells were screened by genomic Southern blot analysis for the appropriate targeting event (Fig. 1). With the occurrence of homologous recombination, the construct was designed to introduce a Bam HI site into the CCK-AR locus. In BamHI-digested mouse genomic DNA, this additional site results in a 6-kb restriction fragment (targeted allele) that can be readily distinguished from the corresponding 10-kb wild-type allele by hybridization analysis (Fig. 1). Positive clones of R1 ES cells were thus identified and cultured in vitro to obtain adequate cell numbers for blastocyst injections.

Generation of CCK-AR ${ }^{-/}$mice. R1 ES cells were injected into blastocysts obtained from C57BL6/J mice (41). Embryos were transferred to pseudopregnant mice. The chimeric male ( $90 \%$ agouti) progeny were bred with $129 / \mathrm{SvEv}$ females. Heterozygous offspring $\left(\mathrm{CCK}-\mathrm{AR}^{+/}\right.$) were identified by genomic Southern blot analysis of Bam HI-digested tail DNA (Fig. 1) and bred to establish the CCK-AR colony. Wild-type and CCK-AR ${ }^{-/-}$breeding pairs, selected from the heterozygous progeny, were bred in parallel to expedite the generation of homozygous mice. The colony of CCK-AR mice was therefore comprised of mice derived from either heterozygous (CCK-AR ${ }^{-/+}$) matings or from homozygous breeding pairs. Experiments were done using age- and sexmatched knockout and wild-type animals. With the exception of the youngest mice included in the age-dependent weight curves (see Fig. 4), all animals studied were at least 7 weeks old.

Pharmacologic confirmation of the absence of functional CCK-A receptors. The density of ${ }^{125}$ I-CCK-8 binding sites was compared between pancreatic membranes isolated from wild-type and CCK-AR ${ }^{-/}$mice. Low-speed supernatant $(600 \mathrm{~g}, 10 \mathrm{~min})$ of pancreatic homogenates was spun at $100,000 \mathrm{~g}$ for $1 \mathrm{~h}$, washed, and resuspended in HBSS supplemented with $1.2 \mathrm{mM}$ EGTA, 4.2 $\mathrm{mM} \mathrm{MgCl}_{2}, 25 \mathrm{mM}$ HEPES (pH 6.5), $150 \mu \mathrm{M}$ PMSF, $250 \mathrm{mg} / \mathrm{ml}$ bacitracin, and $0.2 \%$ BSA. For binding experiments, aliquots of $30 \mu \mathrm{g}$ of protein in $273 \mu \mathrm{l}$ of modified HBSS (see above) were incubated for $100 \mathrm{~min}$ at $37^{\circ} \mathrm{C}$, in the presence of $25 \mathrm{pM}{ }^{125} \mathrm{I}$ CCK-8, with increasing concentrations of unlabeled CCK-8 (up to $1 \mu \mathrm{M}$ ) as competitor. Free radioligand was removed by vacuum filtrating (Whatman GF/B, Whatman, Maidstone, England) at 7 psi and washing with modified HBSS. Bound radioligand 
retained on the filter was determined by $\gamma$ counting (Beckman GAM 5500, Beckman, Fullerton, California, USA). Nonlinear curve fitting was used to determine the density of ${ }^{125}$ I-CCK-8 binding sites, as well as the dissociation constant for CCK-8 (Ligand software, version 4.7 for Macintosh computers, P.J. Munson, National Institutes of Health, Bethesda, Maryland, USA).

Cholecystokinin-induced short-term alteration in food intake. Mice were maintained on a $60: 40$ ( $\mathrm{vol} / \mathrm{vol})$ solution of chocolate-flavored Ensure (Abbott Laboratories, Columbus, Ohio, USA) diluted in water. The liquid diet was presented for $60 \mathrm{~min}$ in the morning (0930-1030) and $90 \mathrm{~min}$ in the afternoon (1530-1700) using 50-ml plastic centrifuge tubes (Nalge Company, Rochester, New York, USA) adapted with leak-proof stainless steel sippers. Water was freely available before and after the feeding periods. Experiments were not initiated until baseline intakes were stable for a period of 7 days.

CCK-AR ${ }^{-/-}(10$ male $)$, CCK-AR ${ }^{+/+}(10$ male $)$, CCK-BR ${ }^{-/-}(5$ male, 5 female), and CCK-BR ${ }^{+/}$(4 male, 5 female) mice were administered intraperitoneal injections of CCK-8, a full agonist at both receptor subtypes (42). Animals were habituated to the procedure with saline injections for at least 2 days before initiation of the experimental protocol. All animals were tested with each dose of CCK-8 $(0,1,10$, and $30 \mu \mathrm{g} / \mathrm{kg}$ body weight) in a counterbalanced order. Experiments were initiated at 0900 after an overnight fast. Total liquid diet intake was measured at 15 and $60 \mathrm{~min}$ after injection. Administration of the different doses of cholecystokinin was separated by at least 5 days.

Conditioned taste aversion. Wild-type (16 male) and CCK-AR ${ }^{-1}$ (16 male) mice were provided free access to $0.15 \%$ saccharin solution for $60 \mathrm{~min}$ (1200-1300); the mice were otherwise saccharin naive. The solution was presented in $50-\mathrm{ml}$ plastic centrifuge tubes (Nalge Company) adapted with leak-proof stainless steel sippers. Within each group (wild-type or mutant), mice were paired based on initial saccharin consumption. Immediately after the initial exposure to saccharin, the paired animals were injected with either $10 \mu \mathrm{g} / \mathrm{kg}$ CCK- 8 or an equivalent volume of $0.9 \%$ saline. Ninety-six hours later, animals were again presented with the saccharin solution. Total fluid intake over a 60 -min period was measured.

\section{Baseline food consumption.}

Comparison of $24-h$ food intakes. Sixteen CCK-A receptor ${ }^{-/}$(8 male, 8 female) and 18 wild-type ( 8 male, 10 female) mice were allowed free access to ground standard Purina rodent laboratory chow (5001, PMI Nutrition International Inc., St. Louis, Missouri, USA) and water. Chow was presented in Wahmann L306A food cups (Wahmann Manufacturing Co., Baltimore, Maryland, USA) fitted with stainless steel screens to prevent spillage. Intake was measured daily for 19 days.

Comparison of food intake during the light vs. the dark cycle. CCK-A receptor $^{--}(n=7)$ and wild-type $(n=8)$ female mice were allowed free access to ground standard Purina rodent laboratory chow and water as described above. Food and fluid intakes were measured every $12 \mathrm{~h}$ at the beginning and end of the light cycle (0800 and 2000) for a period of 14 days.

Weight curves. Weight curves (age in days vs. weight in grams) were determined for CCK-AR ${ }^{-/}$vs. wild-type animals over a period approaching 300 days (see Fig. 4). Mice were freely fed a diet of Pro-Lab rodent chow (R-M-H 3000, Agway Inc., Syracuse, New York, USA). Weight measurements were made using a Mettler BB300 balance (Mettler Toledo Inc., Columbus, Ohio, USA).

Data were obtained using more than 130 animals from each of four groups in the CCK-AR colony: $\mathrm{male}^{-/-}$, $\mathrm{female}^{-/-}$, male wild-type, and female wild-type (see Fig. 4). The weight of each animal was, on average, recorded on 2.1 different days.

Pancreatic histology/immunohistochemistry. Five male, agematched pairs of mice (wild-type and CCK-AR ${ }^{-/}$), between 7 and 17 weeks old, were anesthetized with sodium amytal (Eli Lilly a
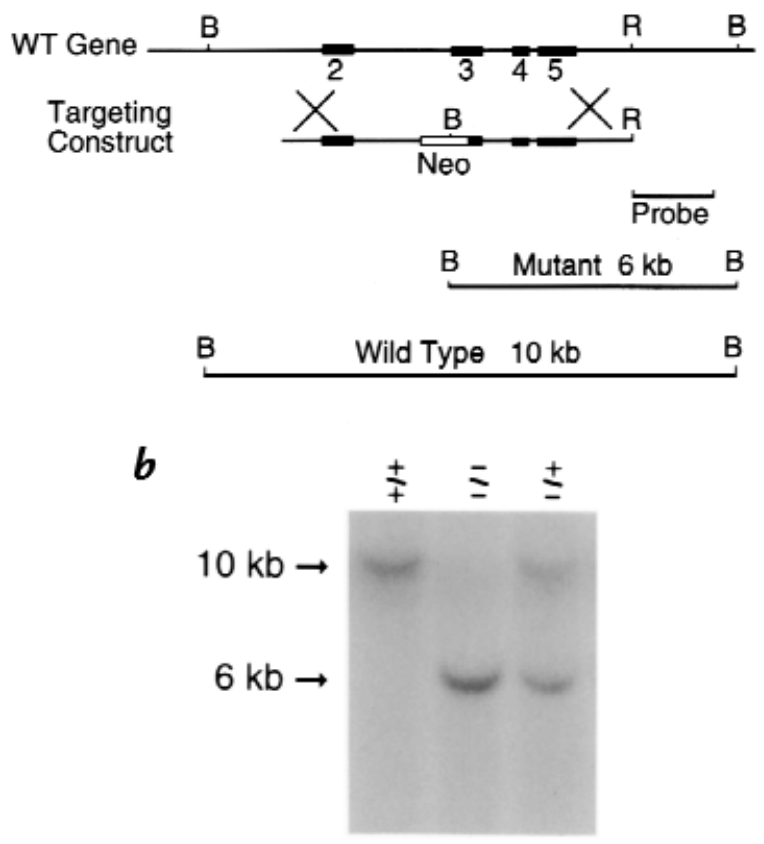

c

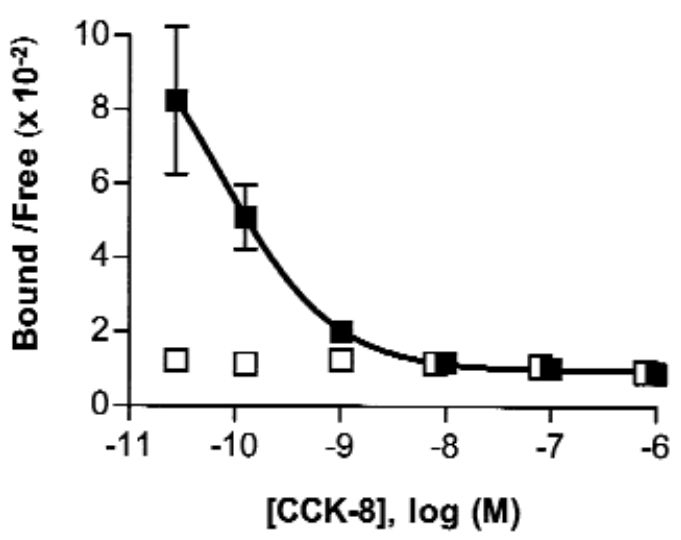

Figure 1

Targeted disruption of the CCK-A receptor gene. (a) Schematic representation comparing the mouse CCK-A receptor gene, the targeting construct, and restriction fragments that distinguish the wild-type $(W T)$ and the disrupted (mutant) CCK-AR alleles. Numbers correspond to exons that were included in the targeting vector. A neomycin resistance gene replaced part of exon 3 and intron 2 as detailed in the text. (b) Genomic Southern blot analysis confirms CCK-AR gene targeting. Mouse-tail DNA was isolated, digested with BamHI, and hybridized to a probe corresponding to CCK-AR gene sequence flanking the $3^{\prime}$ end of the targeting construct. Wild-type $(+/+)$ and mutant $(-/-)$ alleles result in 10- and 6-kb hybridization signals, respectively. (c) CCK-AR ${ }^{-1-}$ mice lack ${ }^{125}$-CCK-8 binding sites in the pancreas. Pancreatic membranes were incubated with 25 pM ${ }^{125}$-CCK-8 in the presence of 0-1 $\mu \mathrm{M}$ unlabeled CCK-8. For Scatchard analysis, tested concentrations of labeled plus unlabeled peptide ( $x$-axis) were plotted against the ratio of bound/free radioligand ( $y$-axis). Data represent mean \pm SEM of three independent experiments. Results of each individual experiment were analyzed by nonlinear curve fitting. CCK-8 binding in pancreatic membranes from wild-type animals (solid squares) had an average $K_{d}$ of $0.18 \mathrm{nM}$ and an average $B_{\max }$ of $101 \mathrm{fmol} / \mathrm{mg}$ protein. In contrast, no displaceable binding was detected in pancreatic membranes from CCK-AR (-/-) mice (open squares). B, Bam HI; Neo, neomycin resistance gene; $R$, EcoRI. 

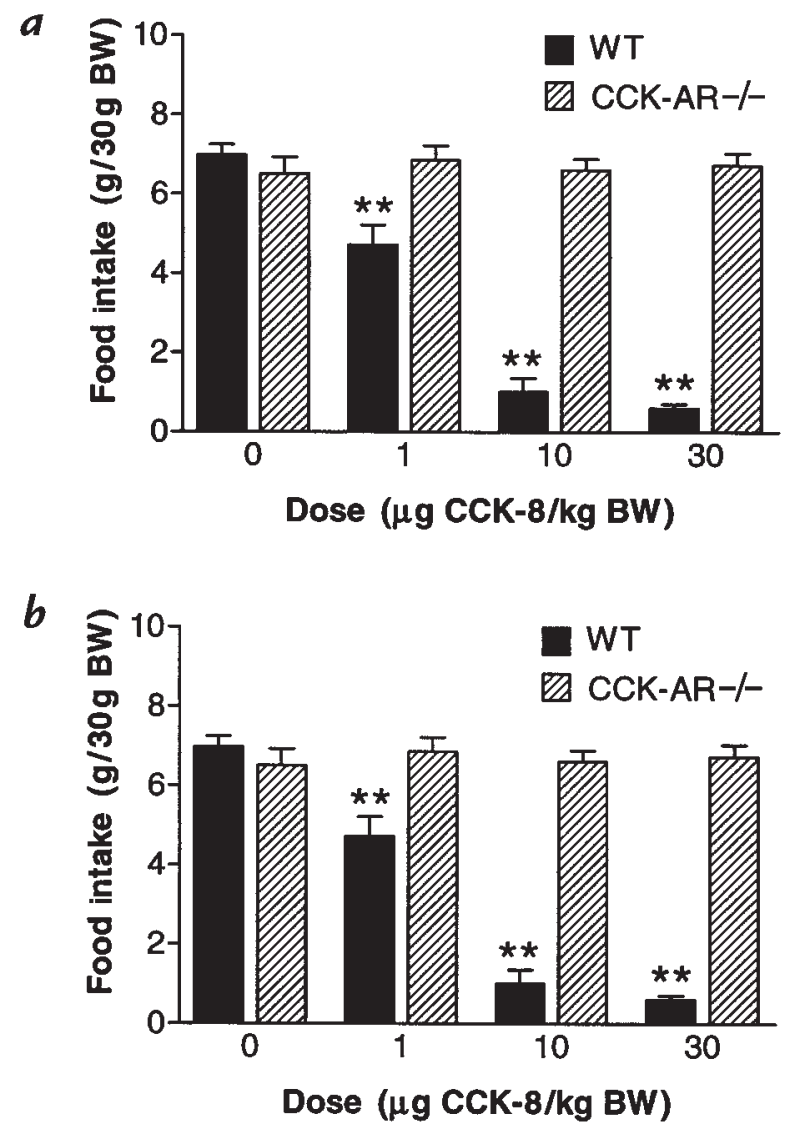

Figure 2

CCK-8 induced inhibition of food intake is mediated through the CCK-A receptor. After an overnight fast, animals were injected with either saline or CCK-8 and provided access to chocolate-flavored Ensure as described in Methods. Cumulative intake (mean \pm SEM) over a 15-min period after injection is shown. Significance vs. intake after saline injection $(0 \mu \mathrm{g}$ CCK- $8 / \mathrm{kg}$ body weight $[B W]$ ): ${ }^{*} P<0.05,{ }^{*} P<0.01$. (a) CCK-8 induced, dose-dependent inhibition of food intake is observed in wild-type (WT), but not in CCK-AR ${ }^{-/-}$mice. Food consumption by 10 wild-type and 10 CCK-AR ${ }^{-/-}$animals was compared. ANOVA parameters were $[\mathrm{F}(3,39)=$ 89.23, $P<0.0001]$ and $[\mathrm{F}(3,39)=0.16, P=0.92]$ for comparisons among wild-type and CCK-AR ${ }^{-1-}$ animals, respectively. $(\boldsymbol{b})$. CCK- 8 induced, dosedependent inhibition of food intake is observed in both wild-type and CCK-BR $-1-$ mice. Food consumption by 9 wild-type and 10 CCK-BR ${ }^{-/-}$animals was compared. ANOVA parameters were $[\mathrm{F}(3,35)=44.40, P<$ $0.0001]$ and $[F(3,39)=135.21, P<0.0001]$ for comparisons among wildtype and CCK-BR $-1-$ animals, respectively. $B W$, body weight.

and Co., Indianapolis, Indiana, USA) and sacrificed. The pancreas was removed, dissected free of lymph nodes and fat, fixed in Bouin's solution, and embedded in paraffin. To qualitatively examine the ratio of $\beta$ to non- $\beta$ cells in the pancreatic islets (43), sections $(5 \mu \mathrm{m})$ were incubated with a mixture of three primary rabbit antibodies directed against different non- $\beta$-cell peptides: (a) bovine glucagon (final dilution 1:3,000; gift of M. Appel, University of Massachusetts Medical School, Worcester, Massachusetts, USA); (b) synthetic somatostatin (final dilution 1:300); and (c) bovine pancreatic polypeptide (final dilution 1:3,000; gift of R. Chance, Eli Lilly and Co.). Bound primary antibodies were detected using a peroxidase-conjugated goat secondary antibody with diaminobenzidine as the chromogen. Sections were counterstained with hematoxylin and eosin.

To assess whether islet fibrosis occurred at older ages, additional pancreatic tissue was analyzed from six male and four female age-matched pairs of mice (wild-type vs. CCK-AR ${ }^{-/}$) ranging in age between 9 and 20 months. After formalin fixation, paraffin-embedded sections were stained with hematoxylin and eosin.

Glucose measurement. Animals were freely fed a diet of Pro-Lab rodent chow. A morning sample of whole blood from mice ranging in age between 7 and 14 months was obtained from the tail. Blood glucose was determined using an automatic glucose monitor (One-Touch Basic; Lifescan Inc., Milpitas, California, USA).

Statistical comparisons. Results shown in Fig. 2 were analyzed using repeated measures ANOVA. Significant differences were further examined with a Tukey HSD post hoc test using the SPSS statistics program (SPSS Inc., Chicago, Illinois, USA). All other statistical comparisons were done using the two-tailed unpaired Student's $t$ test.

\section{Results}

Absence of the CCK-A receptor in homozygous knockout mice. Homozygous CCK-AR ${ }^{-1-}$ mice were generated as detailed in Methods. Genetic ablation of the receptor was demonstrated by genomic Southern blot analysis of tail DNA (Fig. 1). Loss of a 10-kb Bam HI restriction fragment concurrent with the appearance of a 6-kb fragment indicated disruption of both CCK-AR alleles (-/-). To ensure that the receptor in the mutant mice was, in fact, nonfunctional, ${ }^{125}$ I-CCK-8 binding was assessed using pancreatic membranes, one of the major sites of CCK-AR expression in the mouse. ${ }^{125} \mathrm{I}-\mathrm{CCK}-8$ competition-binding experiments (Fig. 1c) revealed that wild-type mouse pancreatic membranes bound CCK- 8 with affinity in the nanomolar range: dissociation constant, $K_{\mathrm{d}}=0.18 \pm 0.03$ $\mathrm{nM}$ (mean \pm SEM, $n=3$ ). In contrast, parallel experiments using membranes isolated from CCK-AR ${ }^{-/-}$mice revealed no displaceable binding of ${ }^{125} \mathrm{I}-\mathrm{CCK}-8$. Thus, by both genetic and pharmacologic criteria, the homozygous knockout mice lack CCK-A receptors.

Cholecystokinin-induced alteration in food intake. After an overnight fast, the effect of CCK-8 administration on food consumption was assessed. Over the 15 -minute period after intraperitoneal injection of CCK-8, a marked dosedependent decrease in total food intake was observed in wild-type animals (Fig. 2, $a$ and b). Compared with intake after saline injection, a significant decrease in feeding resulted from either 1,10 , or $30 \mu \mathrm{g}$ CCK- $8 / \mathrm{kg}$ body weight. When total intake was assessed over the 60 -minute interval after administration of the peptide, the inhibitory effects of CCK- 8 on food intake were less pronounced (data not shown) than during the initial 15-minute period.

In CCK-BR ${ }^{-1}$ animals, the CCK-8 induced inhibition of food intake was similar to the pattern observed in wild-type mice (Fig. 2b). In contrast, the CCK-AR ${ }^{-/}$mice showed no significant inhibition of food intake with increasing doses of intraperitoneal CCK-8 (Fig. 2a).

To determine whether the CCK-AR-mediated decrease in food intake was the result of exogenous CCK producing a noxious stimulus, a conditioned taste aversion (CTA) paradigm was used. The dose of CCK-8 selected for the CTA studies was $10 \mu \mathrm{g} / \mathrm{kg}$ body weight, which led to a near-maximal inhibition of food intake in wild-type mice (Fig. 2a). There was no significant difference in saccharin consumption when compared before and after conditioning with either saline or CCK-8 (Table 1). This pattern of response suggests that CCK-8, at the tested 
dose, is nonaversive. As expected, CCK-AR knockout animals showed no apparent aversion to saccharin after conditioning with CCK-8 (data not shown).

Baseline food intake. There was no significant difference in 24-hour baseline food or water intake between wildtype and CCK-AR ${ }^{-/}$animals maintained on a free-feeding schedule (Fig. 3a). In addition, when food consumption was examined during the light vs. the dark cycle, both wild-type and CCK-AR ${ }^{-1-}$ mice showed a normal diurnal variation, with the majority of food intake occurring during the dark cycle (Fig. 3b). Furthermore, there was no significant difference between the intakes of wild-type and knockout mice during either the light or the dark cycle.

Long-term study of body weight. For the CCK-AR mouse colony, the respective growth curves (age vs. weight) of sex-matched wild-type and receptor ${ }^{-}$- animals are essentially superimposable (Fig. 4). Male and female mice were analyzed independently, because male mice of each genotype (wild-type and -/-) were heavier than corresponding age-matched females (compare Fig. 4, a vs. b).

Pancreatic morphology and glucose homeostasis. Additional studies were performed to determine whether abnormalities in pancreatic morphology (i.e., islet fibrosis), which have been observed in OLETF rats $(31,44-46)$, are also present in CCK-AR ${ }^{-/-}$mice. Representative pancreatic sections from CCK-AR ${ }^{-/-}$and wild-type mice, ranging in age between 7 and 17 weeks, are compared in Fig. $5 a$. Both the acinar gland and islet morphology were comparable in knockout and wild-type mice. There was no evidence of fibrosis or differences in the $\beta$ - to non- $\beta$ cell ratio. To determine if islet fibrosis occurred in older mice, the pancreata of an additional group of six male and four female age-matched pairs of mice (wild-type and $\mathrm{CCK}-\mathrm{AR}^{-1}$ ), ranging in age from 9 to 21 months, were also examined. There was no evidence of islet fibrosis in any of these older mice (not shown).

It has been reported that male OLETF rats develop diabetes with aging $(31,32)$. In contrast, blood glucose levels of CCK-AR ${ }^{-1-}$ mice (7-13 months) on a freely fed diet were not significantly different from the corresponding values of age- and sex-matched wild-type controls (Fig. 5b). Normal blood glucose levels were also observed in male and female CCK-BR ${ }^{-1-}$ animals (8-14 months) when compared with wild-type age-matched controls (data not shown).

\section{Discussion}

In this study we report the generation of CCK-AR $-1-$ mice by targeted gene disruption. The CCK- $\mathrm{AR}^{-/-}$animals are viable, fertile, and have now been observed for periods

\section{Table 1}

Cholecystokinin-8 does not result in conditioned taste aversion.

\begin{tabular}{ccc}
\hline Saccharin intake (Pre) & Conditioning & Saccharin intake (Post) \\
$1.77 \pm 0.14$ & Saline & $2.12 \pm 0.18$ \\
$2.17 \pm 0.20$ & CCK- 8 & $2.21 \pm 0.17$ \\
\hline
\end{tabular}

Sixteen wild-type male mice were divided into two groups. Intake of saccharinsweetened water (pre) was measured in grams (mean \pm SEM, $n=8$ ). One group of animals was conditioned with saline, the other with CCK-8. Saccharin-flavored water intakes were repeated after 96 hours (post). There was no significant difference in intakes after conditioning $[\mathrm{t}(14)=-0.35, P=0.73]$.
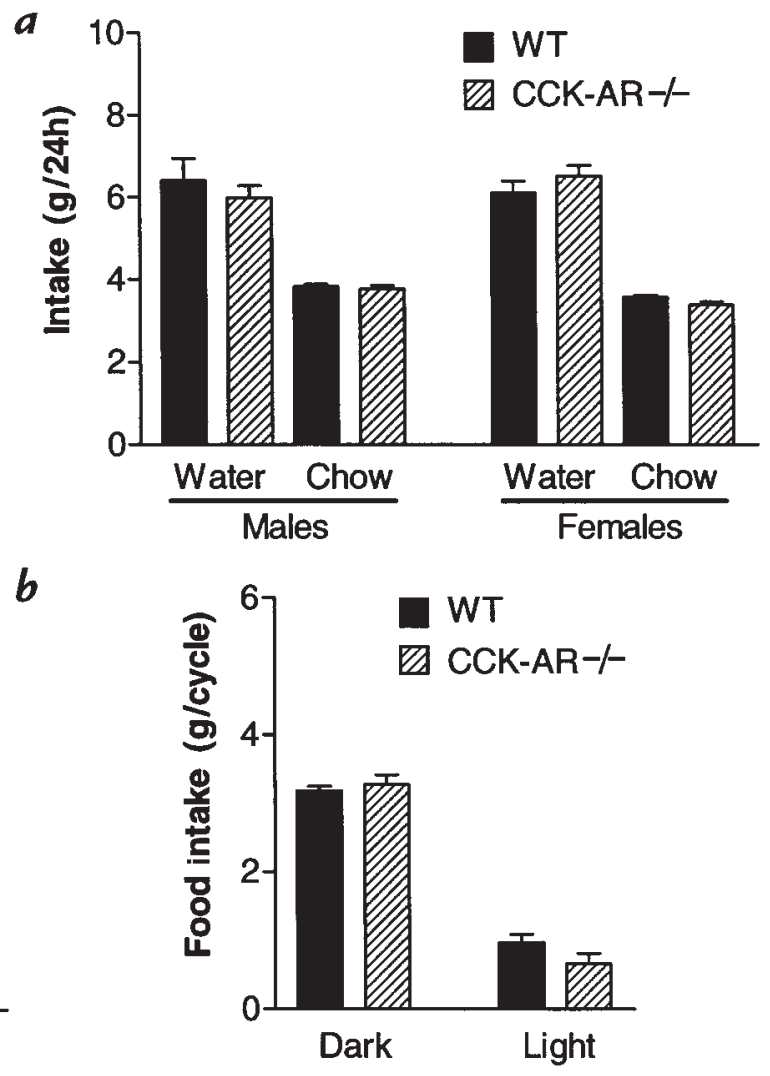

Figure 3

Food and water intake is normal in freely fed CCK-AR ${ }^{-1-}$ mice $(\boldsymbol{a})$ Twenty-four hour intake of water and Purina rodent laboratory chow are not significantly different $(P>0.05)$ between wild-type and CCK-AR ${ }^{-/}$mice that were fed ad libitum. Average daily intake (mean \pm SEM) over a 19-day period is shown.

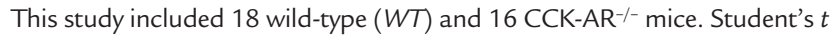
values for comparison between wild-type and CCK-AR $-1-$ mice were: $t(14)=$ $0.69, P=0.50$ (males, water); $t(14)=0.59, P=0.57$ (males, chow); $t(16)=$ $-1.02, P=0.32$ (females, water); and $t(16)=1.95, P=0.07$ (females, chow). (b) Normal diurnal variation in food intake of CCK-AR ${ }^{-/-}$mice. Eight wildtype and seven CCK-AR ${ }^{-/-}$mice were allowed ad libitum access to Purina rodent laboratory chow. Average intake of chow (mean \pm SEM) during the light and dark cycles, each $12 \mathrm{~h}$ long, was recorded over a 14-day period. There was no significant difference $(P>0.05)$ between the intake of wild-type $(W T)$ and $C C K-A R^{-/-}$mice during either the light or the dark period. Student $t$ values for comparison between wild-type and CCK-AR-1- mice were: $t(13)=$ $-0.74, P=0.48$ (food intake, dark); $t(13)=1.57, P=0.14$ (food intake, light).

exceeding 12 months. Although the animals are prone to the development of gallstones (47), their appearance and general behavior is comparable to that of corresponding wild-type controls. The CCK-AR $-1-$ mice and previously reported CCK-BR $-/-$ mice $(33,34)$ provide genetically defined models for examining the wide spectrum of physiological functions that are postulated to be modulated by cholecystokinin (9).

The availability of receptor knockout mice allowed us to examine the role of the CCK-AR and the CCK-BR in modulating food intake without the potential confounding variables that may arise from pharmacologic and/or surgical manipulations. When wild-type mice were observed after an intraperitoneal injection of CCK-8, a dose-dependent decrease in food intake was evident (Fig. 2). As suggested by the conditioned taste aver- 


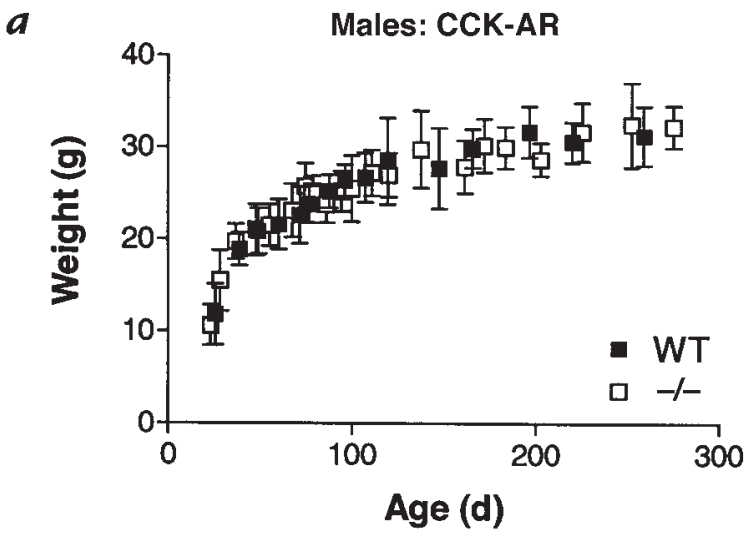

$\boldsymbol{b}$

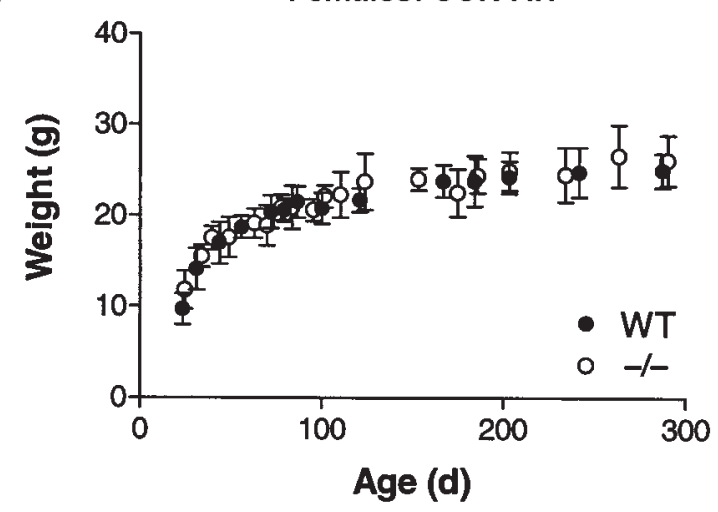

Figure 4

Weight gain of CCK-AR $-1-$ mice is comparable to that of age- and sexmatched wild-type controls. Mice were freely fed a diet of Pro-Lab rodent chow. Weight measurements of all mice within a given population were sorted by corresponding animal ages, and then pooled into groups comprised of 15 consecutive data points each. Average ages within these groups are plotted vs. the mean \pm SD of corresponding weights. (a) Body weights of male CCK-AR ${ }^{-/-}$mice show no difference from respective values of wild-type (WT) controls; 171 mutant and 151 wild-type animals were compared. $(\boldsymbol{b})$ Body weights of female CCK-AR ${ }^{-/}$mice show no difference from respective values of wild-type controls; 156 mutant and 133 wild-type animals were compared.

sion paradigm (Table 1), the inhibitory effect of CCK-8 in the mice is unlikely to be the result of an aversive stimulus (e.g., nausea). The finding that CCK-8 is nonaversive at doses that inhibit food intake is consistent with multiple reports in the literature $(1,48-51)$ and further supports the view that CCK-8 triggers a physiologic satiety signal. When CCK-BR $-1-$ mice were studied, the dosedependent CCK-8-induced inhibition of food intake resembled the pattern observed in wild-type animals. The latter finding suggests that the CCK-BR is not involved in mediating the inhibitory effect of exogenous CCK.

In contrast to both wild-type animals and those lacking the CCK-BR, CCK-AR $-/$ mice show no change in food consumption after administration of exogenous CCK-8. These observations support the conclusion that CCK-induced inhibition of food intake is mediated through the CCK-AR. Our findings are in agreement with a large body of evidence that demonstrates that CCK-AR-specific antagonists block the effects of periph- erally administered CCK on food consumption, whereas CCK-BR antagonists do not (7-9). In addition, multiple studies examining the effectiveness of CCK/gastrin peptides in modulating feeding behavior suggest a rank order of agonist potencies that is consistent with a CCKAR- rather than a CCK-BR-mediated behavior (7-9).

Based on reports of both short-term inhibition of food intake with exogenous CCK and increased feeding in response to CCK-receptor antagonists, there has been speculation regarding the role of endogenous cholecystokinin in the maintenance of body weight (9). Studies with exogenous CCK-8, administered either continuously or intermittently, failed to produce weight loss over either a 14- or 6-day period, respectively $(52,53)$. However, the relatively short duration of these studies, together with issues of ligand administration (e.g., dosing, timing, bioavailability) and receptor desensitization limit the interpretation of these experiments. Given these constraints, it would be even more difficult to use long-term application of antagonists as a strategy for investigating the role of endogenous CCK in modulating body weight. The availability of mice lacking either CCK-A or CCK$\mathrm{B} /$ gastrin receptors provided an alternative approach to address this question without the necessity of pharmacologic manipulations.

To examine the physiologic role of endogenous CCK over an extended period of time, weight as a function of age was assessed in wild-type vs. knockout mice that were fed ad libitum (Fig. 4). CCK-AR-/- mice had body weights comparable to the corresponding age- and sexmatched wild-type controls. The similarity in weights between $\mathrm{CCK}-\mathrm{AR}^{-/}$and wild-type animals persisted through the rapid growth phase and extended well into adulthood (Figs. 4, $a$ and $b$ ). A parallel analysis of CCK$\mathrm{BR}^{-/-}$mice revealed that these animals also reached adult weights that were indistinguishable from the corresponding age- and sex-matched controls (data not shown). Taken together, these data indicate that neither the CCK-AR nor the CCK-BR is essential for the maintenance of normal body weight.

In light of the extensive literature implicating CCK in satiety, it is somewhat surprising that absence of the CCK regulatory pathway does not result in obesity. It is possible that the CCK-AR only plays a role in mediating short-term food intake and that the mechanisms regulating body weight are distinct. Analogous to CCK, both glucagon-like peptide 1 and neuropeptide $Y$ have been demonstrated to modulate food intake when exogenously administered $(26,54,55)$, yet disruption of the respective receptor or peptide did not result in an alteration in body weight $(27,28)$. CCK may therefore join a growing list of peptide hormones (e.g., glucagon-like peptide 1 , neuropeptide $Y$ ) that appear to modulate short-term food intake yet are not essential for the maintenance of body weight.

An alternative explanation for the absence of obesity in the CCK-AR ${ }^{-/}$mice is that other mechanisms known to play a role in the control of satiety and/or body weight, provide functional redundancy (54-56). Candidate pathways that could potentially compensate for the loss of the cholecystokininergic system might include a range 

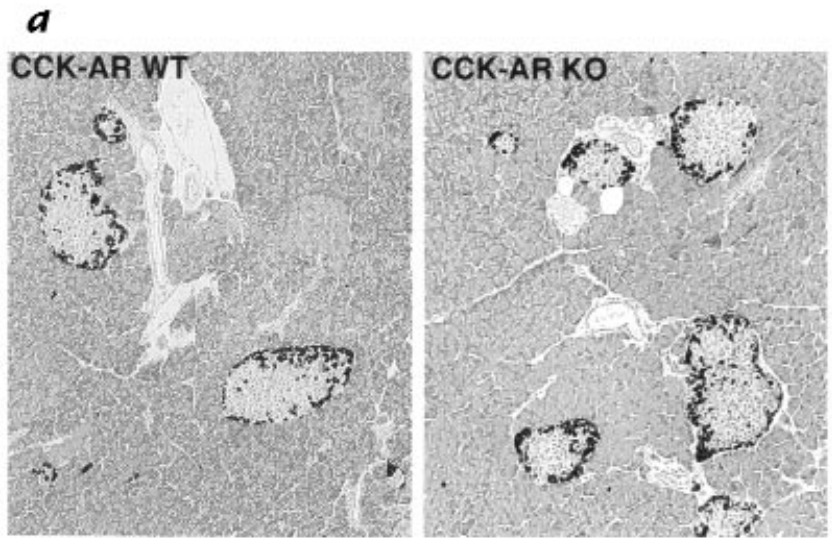

$b$

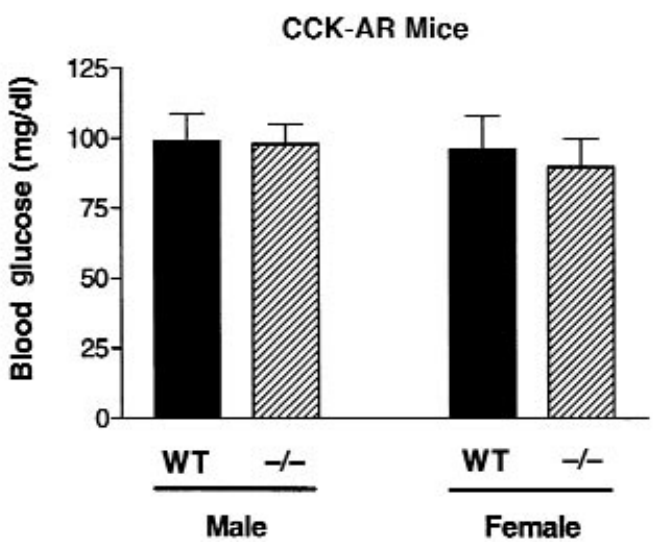

Figure 5

$\mathrm{CCK}-\mathrm{AR}^{-1-}$ mice have normal pancreatic histology and blood glucose levels (a) Normal histology of exocrine and endocrine pancreatic tissue from $C \mathrm{CK}-\mathrm{AR}^{-/-}$mice. Non- $\beta$ islet cells were immunostained; sections were counterstained with hematoxylin and eosin. Both the islet and acinar gland morphology are comparable in wild-type (WT) and CCK$\mathrm{AR}^{-1-}$ (knockout, or $\left.K O\right)$ mice (see text). The pancreatic histology that is shown is representative of sections from five age-matched pairs (WT and $\mathrm{CCK}-\mathrm{AR}^{-1-}$ ) of male mice. (b) Blood glucose levels in CCK-AR ${ }^{-1-}$ mice are not significantly different from the respective values of ageand sex-matched wild-type controls. Morning glucose levels were determined in freely fed mice. Average age and number of animals in the corresponding groups were as follows: $311 \pm 53$ days ( 12 wild-type males);

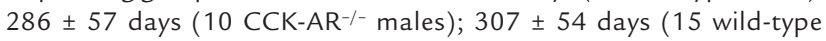
females); $330 \pm 54$ days (14 CCK-AR ${ }^{-1-}$ females). Data are given as mean \pm SD. Student's $t$ values for glucose comparisons between wildtype and CCK-AR ${ }^{-/-}$mice were: $t(20)=0.27, P=0.79$ (males); $t(27)=$ $1.46, P=0.16$ (females).

of central mediators (e.g., serotonin, neuropeptide Y, orexins, corticotropin-releasing hormone), as well as a number of peripheral peptides (e.g., leptin, gastrin-releasing peptide, and glucagon-like peptide 1) (54, 55, 57-59).

A line of rats, Otsuka Long-Evans Tokushima Fatty (OLETF), which was initially developed as a model of non-insulin-dependent diabetes mellitus $(31,44)$, has recently been shown to lack CCK-A receptors because of a spontaneous deletion in the corresponding receptor gene $(29,30)$. Comparison between the CCK-AR $\mathrm{AR}^{-1-}$ mice and the OLETF rats reveals several important differences. The OLETF rats have increased body weight, are prone to diabetes mellitus, and develop pancreatic islet fibrosis with age relative to wild-type strains (31, 44-46). In contrast, we have shown that the $\mathrm{CCK}-\mathrm{AR}^{-/-}$mice maintain normal weight, normal blood glucose levels, and have pancreatic islet morphology that is indistinguishable from wild-type controls.

The apparent discrepancy in phenotypes between OLETF rats and the CCK-AR knockout mice may be explained by interspecies differences (e.g., tissue distribution of receptors) that influence how absence of the CCK-AR manifests as physiologic alterations. Expression of CCK-A receptors within the brain and spinal cord is highly species dependent (60-64) and could thus potentially influence the role of this receptor in the regulation of food intake. Alternatively, species differences in pathways which may interact with the cholecystokininergic system in determining body weight (e.g., serotonin, leptin, and gastrin-releasing peptide) (55) could indirectly affect the phenotypic consequences of CCK-AR absence.

Finally, it must also be considered that obesity, diabetes, and islet fibrosis in the OLETF strain may be the result of additional, as yet undefined, genetic alterations in the rats. This possibility has been suggested based on the segregation of phenotypic markers within the OLETF lineage, as well as linkage analysis on offspring of OLETF rats crossed with normoglycemic strains $(31,32)$. The diabetic phenotype of the OLETF rats is likely the product of multiple interacting genes that may include the CCK-AR and ODB1 genes (32). Furthermore, the occurrence of pancreatic islet fibrosis has been observed in rat strains with no apparent defect in the CCK-AR (65, 66). It therefore remains to be explored to what extent the range of abnormalities observed in OLETF rats is explained by gene defects that are unrelated to absence of functional CCK-A receptors.

One advantage of studying animals generated by targeted gene disruption is that the resulting phenotype can be specifically attributed to the absence of a single gene product. At least in mice, our findings suggest that endogenous CCK is not essential for the long-term maintenance of body weight or euglycemia. Still, our findings cannot exclude that CCK may be required to prevent obesity or hyperglycemia in other species with a genetic background and/or compensatory mechanisms that are considerably different from those of mice.

\section{Acknowledgments}

We thank A. Leiter, A. Kane, S. Krasinski, and K. Schaffer for careful reading of the manuscript, and C. Chen, B. Desai, M. Chiu, and M. Taneja for technical assistance. This work was supported by the National Institutes of Health grants NIDDK46767 (to A. Kopin), NIDDK44523 (to S. BonnerWeir), NIDA04132 (to R. Kanarek), an American Home Products grant (to A. Kopin), an ADHF/AGA Industry Research Scholar Award (to M. Beinborn), and a DFG grant (to F. Schmitz). A. Kopin is a New England Medical Center MCRI investigator. Part of this work was performed in the Molecular Biology and Microbiology Core facilities of the GRASP Digestive Disease Center (New England Medical Center, Tufts University School of Medicine, Boston, Massachusetts, USA) (P30-DK34928). 
1. Gibbs, J., Young, R.C., and Smith, G.P. 1973. Cholecystokinin decreases food intake in rats. J. Comp. Physiol. Psychol. 84:488-95.

2. Himick, B.A., and Peter, R.E. 1994. CCK/gastrin-like immunoreactivity in brain and CCK suppression of feeding in goldfish. Am. J. Physiol. 267:R841-R851.

3. Khosla, S., and Crawley, J.N. 1988. Potency of L-364,718 as an antagonist of the behavioral effects of peripherally administered cholecystokinin. Life Sci. 42:153-159.

4. Hirosue, Y., et al. 1993. Cholecystokinin octapeptide analogues suppress food intake via central CCK-A receptors in mice. Am. J. Physiol. 265:R481-R486.

5. Asin, K.E., Bednarz, L., Nikkel, A.L., Gore, P.A., Jr., and Nadzan, A.M 1992. A-71623, a selective CCK-A receptor agonist, suppresses food intake in the mouse, dog, and monkey. Pharmacol. Biochem. Behav. 42:699-704.

6. Baldwin, B.A. 1992. CCK as a putative satiety factor in farm animals. In Multiple cholecystokinin receptors in the CNS. C.T. Dourish, S.J. Cooper, S.D. Iversen, and L.L. Iversen, editors. Oxford University Press. New York, NY. 206-221.

7. Dourish, C.T. 1992. Behavioral analysis of the role of CCK-A and CCK$\mathrm{B}$ receptors in the control of feeding in rodents. In Multiple cholecystokinin receptors in the CNS. C.T. Dourish, S.J. Cooper, S.D. Iversen, and L.L. Iversen editors. Oxford University Press. New York, NY. 234-253.

8. Smith, G.P., and Gibbs, J. 1992. The development and proof of the CCK hypothesis of satiety. In Multiple cholecystokinin receptors in the CNS. C.T. Dourish, S.J. Cooper, S.D. Iversen, and L.L. Iversen, editors. Oxford University Press. New York, NY. 166-182.

9. Crawley, J.N., and Corwin, R.L. 1994. Biological actions of cholecystokinin. Peptides. 15:731-755

10. Jensen, R. T., et al. 1994. Distinguishing multiple CCK receptor subtypes. Studies with guinea pig chief cells and transfected human CCK receptors. Ann. NY Acad. Sci. 713:88-106.

11. Hewson, G., Leighton, G.E., Hill, R.G., and Hughes, J. 1988. The cholecystokinin receptor antagonist L364,718 increases food intake in the rat by attenuation of the action of endogenous cholecystokinin. Br. J. Pharmacol. 93:79-84.

12. Reidelberger, R.D., and O'Rourke, M.F. 1989. Potent cholecystokinin antagonist L-364,718 stimulates food intake in rats. Am. J. Physiol. 257:R1512-R1518

13. Moran, T.H., Ameglio, P.J., Peyton, H.J., Schwartz, G.J., and McHugh, P.R 1993. Blockade of type A, but not type B, CCK receptors postpones satiety in rhesus monkeys. Am. J. Physiol. 265:R620-R624.

14. Wolkowitz, O.M., et al. 1990. Hunger in humans induced by MK-329, a specific peripheral-type cholecystokinin receptor antagonist. Biol. Psychiatry. 28:169-173

15. Gregory, P.C., McFadyen, M., and Rayner, D.V. 1989. Duodenal infusion of fat, cholecystokinin secretion and satiety in the pig. Physiol. Behav. 45:1021-1024.

16. Cooper, S.J., Dourish, C.T., and Barber, D.J. 1990. Reversal of the anorectic effect of $(+)$-fenfluramine in the rat by the selective cholecystokinin receptor antagonist MK-329. Br. J. Pharmacol. 99:65-70.

17. Dourish, C.T., Rycroft, W., and Iversen, S.D. 1989. Postponement of satiety by blockade of brain cholecystokinin (CCK-B) receptors. Science. 245:1509-1511.

18. Reidelberger, R.D., Varga, G., and Solomon, T.E. 1991. Effects of selective cholecystokinin antagonists L364,718 and L365,260 on food intake in rats. Peptides. 12:1215-1221.

19. Corwin, R.L., Gibbs, J., and Smith, G.P. 1991. Increased food intake after type A but not type B cholecystokinin receptor blockade. Physiol. Behav. 50:255-258.

20. Weatherford, S.C., Chiruzzo, F.Y., and Laughton, W.B. 1992. Satiety induced by endogenous and exogenous cholecystokinin is mediated by CCK-A receptors in mice. Am. J. Physiol. 262:R574-R578.

21. Moran, T.H., Ameglio, P.J., Schwartz, G.J., and McHugh, P.R. 1992. Blockade of type A, not type B, CCK receptors attenuates satiety actions of exogenous and endogenous CCK. Am.J. Physiol. 262:R46-R50.

22. Susulic, V.S., et al. 1995. Targeted disruption of the beta 3-adrenergic receptor gene. J. Biol. Chem. 270:29483-29492.

23. Tecott, L.H., et al. 1995. Eating disorder and epilepsy in mice lacking 5 HT2c serotonin receptors. Nature. 374:542-546.

24. Ohki-Hamazaki, H., et al. 1997. Mice lacking bombesin receptor subtype3 develop metabolic defects and obesity. Nature. 390:165-169.

25. Huszar, D., et al. 1997. Targeted disruption of the melanocortin-4 receptor results in obesity in mice. Cell. 88:131-141.

26. Turton, M.D., et al. 1996. A role for glucagon-like peptide-1 in the central regulation of feeding. Nature. 379:69-72.

27. Scrocchi, L.A., et al. 1996. Glucose intolerance but normal satiety in mice with a null mutation in the glucagon-like peptide 1 receptor gene. Nat. Med. 2:1254-1258.

28. Erickson, J.C., Clegg, K.E., and Palmiter, R.D. 1996. Sensitivity to leptin and susceptibility to seizures of mice lacking neuropeptide Y. Nature. 381:415-421
29. Funakoshi, A., et al. 1995. An animal model of congenital defect of gene expression of cholecystokinin (CCK)-A receptor. Biochem. Biophys. Res. Commun. 210:787-796.

30. Takiguchi, S., et al. 1997. Disrupted cholecystokinin type-A receptor (CCKAR) gene in OLETF rats. Gene. 197:169-175.

31. Kawano, K., et al. 1992. Spontaneous long-term hyperglycemic rat with diabetic complications. Otsuka Long-Evans Tokushima Fatty (OLETF) strain. Diabetes. 41:1422-1428.

32. Takiguchi, S., et al. 1998. A disrupted cholecystokinin A receptor gene induces diabetes in obese rats synergistically with ODB1 gene. Am. J. Physiol. 274:E265-E270.

33. Langhans, N., et al. 1997. Abnormal gastric histology and decreased acid production in cholecystokinin-B/gastrin receptor-deficient mice. Gastroenterology. 112:280-286.

34. Nagata, A., et al. 1996. G protein-coupled cholecystokinin-B/gastrin receptors are responsible for physiological cell growth of the stomach mucosa in vivo. Proc. Natl. Acad. Sci. USA. 93:11825-11830.

35. de Weerth, A., Pisegna, J.R., Huppi, K., and Wank, S.A. 1993. Molecular cloning, functional expression and chromosomal localization of the human cholecystokinin type A receptor. Biochem. Biophys. Res. Commun. 194:811-818.

36. Ulrich, C.D., et al. 1993. Molecular cloning and functional expression of the human gallbladder cholecystokinin A receptor. Biochem. Biophys. Res. Commun. 193:204-211.

37. Lacourse, K.A., Lay, J.M., Swanberg, L.J., Jenkins, C., and Samuelson, L.C. 1997. Molecular structure of the mouse CCK-A receptor gene. Biochem. Biophys. Res. Commun. 236:630-635.

38. Takata, Y., Takiguchi, S., Funakoshi, A., and Kono, A. 1995. Gene structure of rat cholecystokinin type-A receptor. Biochem. Biophys. Res. Commun. 213:958-966.

39. Baldwin, J.M. 1993. The probable arrangement of the helices in $G$ protein-coupled receptors. EMBO J. 12:1693-1703.

40. Nagy, A., Rossant, J., Nagy, R., Abramow-Newerly, W., and Roder, J.C. 1993. Derivation of completely cell culture-derived mice from early-passage embryonic stem cells. Proc. Natl. Acad. Sci. USA. 90:8424-8428.

41. Manjunath, N., Correa, M., Ardman, M., and Ardman, B. 1995. Negative regulation of $\mathrm{T}$-cell adhesion and activation by CD43. Nature. 377:535-538

42. Liddle, R.A. 1994. Cholecystokinin. In Gut peptides. J.H. Walsh and G.J. Dockray, editors. Raven Press. New York, NY. 175-216.

43. Bruning, J.C., et al. 1997. Development of a novel polygenic model of NIDDM in mice heterozygous for IR and IRS-1 null alleles. Cell. 88:561-572

44. Ishida, K., Mizuno, A., Sano, T., Shi, K., and Shima, K. 1993. Plasma glucagon responses to insulin-induced hypoglycemia and arginine in spontaneous non-insulin-dependent diabetes mellitus (NIDDM) rats, Otsuka Long Evans Tokushima Fatty (OLETF) strain. Acta Endocrinol. 129:585-593.

45. Miyasaka, K., et al. 1994. Lack of satiety effect of cholecystokinin (CCK) in a new rat model not expressing the CCK-A receptor gene. Neurosci. Lett. 180:143-146.

46. Funakoshi, A., et al. 1996. Pancreatic endocrine dysfunction in rats not expressing the cholecystokinin-A receptor. Pancreas. 12:230-236.

47. Schmitz, F., et al. 1996. CCK-A receptor deficient mice have increased susceptibility to cholesterol gallstones. Hepatol. 24:246A. (Abstr.)

48. Kissileff, H.R., Pi-Sunyer, F.X., Thornton, J., and Smith, G.P. 1981. C-terminal octapeptide of cholecystokinin decreases food intake in man. Am. J. Clin. Nutr. 34:154-160.

49. Ervin, G.N., and Teeter, M.N. 1986. Cholecystokinin octapeptide and lithium produce different effects on feeding and taste aversion learning. Physiol. Behav. 36:507-512.

50. West, D.B., Greenwood, M.R., Marshall, K.A., and Woods, S.C. 1987. Lithium chloride, cholecystokinin and meal patterns: evidence that cholecystokinin suppresses meal size in rats without causing malaise. Appetite. 8:221-227.

51. Lieverse, R.J., Jansen, J.B., Masclee, A.M., and Lamers, C.B. 1994. Satiety effects of cholecystokinin in humans. Gastroenterology. 106:1451-1454.

52. Crawley, J.N., and Beinfeld, M.C. 1983. Rapid development of tolerance to the behavioural actions of cholecystokinin. Nature. 302:703-706.

53. West, D.B., Fey, D., and Woods, S.C. 1984. Cholecystokinin persistently suppresses meal size but not food intake in free-feeding rats. Am. J. Physiol. 246:R776-R787.

54. Naggert, J., Harris, T., and North, M. 1997. The genetics of obesity. Curr. Opin. Genet. Dev. 7:398-404.

55. Rosenbaum, M., Leibel, R.L., and Hirsch, J. 1997. Obesity. N. Engl. J. Med. 337:396-407.

56. Nowak, M.A., Boerlijst, M.C., Cooke, J., and Smith, J.M. 1997. Evolution of genetic redundancy. Nature. 388:167-171.

57. Sakurai, T., et al. 1998. Orexins and orexin receptors: a family of hypothalamic neuropeptides and $G$ protein-coupled receptors that regulate feeding behavior. Cell. 92:573-585. 
58. Hampton, L. L., et al. 1998. Loss of bombesin-induced feeding suppression in gastrin-releasing peptide receptor-deficient mice. Proc. Natl. Acad. Sci. USA. 953:188-192.

59. Flier, J.S., and Maratos-Flier, E. 1998. Obesity and the hypothalamus: novel peptides for new pathways. Cell. 92:437-440.

60. Williams, J.A., Gryson, K.A., and McChesney, D.J. 1986. Brain CCK receptors: species differences in regional distribution and selectivity. Peptides. 7:293-296.

61. Sekiguchi, R., and Moroji, T. 1986. A comparative study on characterization and distribution of cholecystokinin binding sites among the rat, mouse and guinea pig brain. Brain Res. 399:271-281.

62. Hill, D.R., Shaw, T.M., and Woodruff, G.N. 1987. Species differences in the localization of 'peripheral' type cholecystokinin receptors in rodent brain. Neurosci. Lett. 79:286-289.

63. Miceli, M.O., and Steiner, M. 1989. Novel localizations of central- and peripheral-type cholecystokinin binding sites in Syrian hamster brain as determined by autoradiography. Eur. J. Pharmacol. 169:215-224.

64. Hill, D.R., and Woodruff, G.N. 1990. Differentiation of central cholecystokinin receptor binding sites using the non-peptide antagonists MK329 and L-365,260. Brain Res. 526:276-283.

65. Reaven, E.P., and Reaven, G.M. 1981. Structure and function changes in the endocrine pancreas of aging rats with reference to the modulating effects of exercise and caloric restriction. J. Clin. Invest. 68:75-84.

66. Wank, S. A., et al. 1992. Purification, molecular cloning, and functional expression of the cholecystokinin receptor from rat pancreas. Proc. Natl. Acad. Sci. USA. 89:3125-3129. 